\title{
VLBI-observations of Solar Wind Plasma by the method of radio raying; theory and experiments
}

\author{
M. B. Nechaeva ${ }^{1} \dagger$, V. G. Gavrilenko ${ }^{2}$, Yu. N. Gorshenkov ${ }^{3}$, \\ B. N. Lipatov ${ }^{1}$, \\ Liu Xiang $^{4}$, I. E. Molotov ${ }^{5}$, A. B.Pushkarev ${ }^{5}$, R. Shanks ${ }^{6}$ \\ ${ }^{1}$ Radiophysical Research Institute, Nizhnii Novgorod, Russia email: nech@nirfi.sci-nnov.ru \\ ${ }^{2}$ Nizhniy Novgorod State University,Nizhniy Novgorod, Russia \\ ${ }^{3}$ Special Research Bureau, Moscow, Russia \\ ${ }^{4}$ Urumqi Astronomical Observatory, Urumqi, China \\ ${ }^{5}$ Central (Pulkovo) Astronomical Observatory, St.-Petersburg, Russia \\ ${ }^{6}$ Dominion Radio Astrophysics Laboratory, Penticton, Canada
}

We represent results of experiments on investigation of solar wind plasma by the method of radio sounding, when the signal from the radio source paths through the solar wind plasma and then it's received at ground-based radiointerferometer.

Heterogeneities of electron density of propagation media cause fluctuations of the received emission and distort the output signal of the instrument. The analysis of these disturbances allows to get information on physical characteristics of the propagation media.

At using of VLBI the signals from the sources to separated antennas go through plasma by different paths. Relative fluctuations, arising at each trajectories, influent at the interferometer signal.

The baseline projection onto the wavefront $(100 \mathrm{~km}-10000 \mathrm{~km})$ determines the maximal heterogeneities' scale, to which the interferometer is sensitive. Using interferometric complexes with various orientation of baseline projections allow to obtain information about spatial structure of solar wind plasma parameters. The observation of sources, located at different angular distances and position angles from the Sun let us to make certain conclusion about anisotropy of electron density of investigated medium.

We carried out the analysis of international VLBI experiments on investigations of solar wind plasma, implemented in 1998, 1999, 2000. At the course of these experiments VLBI complex received the extragalactic source radio emission (wavelength 18 $\mathrm{cm}$ ) passed through the turbulent plasma of solar wind. Radio sources were located at different angular distances from the Sun (4-37 degrees). These observations were realized with participation of radio telescopes, included at Low Frequency VLBI Network: Bear Lakes (RT-64, Russia), Puschino (RT-22, Russia), Hartebeesthock (RT-25, South Africa), Arecibo (RT-305, USA), Urumqi (RT-25, China), Noto (RT-25, Italy), Shanghai (RT-25, China), GMRT (RT-45, India). Preprocessing was carried out with using of S2 correlator at Penticton (Canada).

We performed the post processing of experimental data. The results of spectral analysis of series realization were compared with theoretical conclusions, obtained before $[1,2]$.

$\dagger$ Present address: NIRFI, 25 B. Pecherskaya st., Nizhny Novgorod, 603950, Russia 


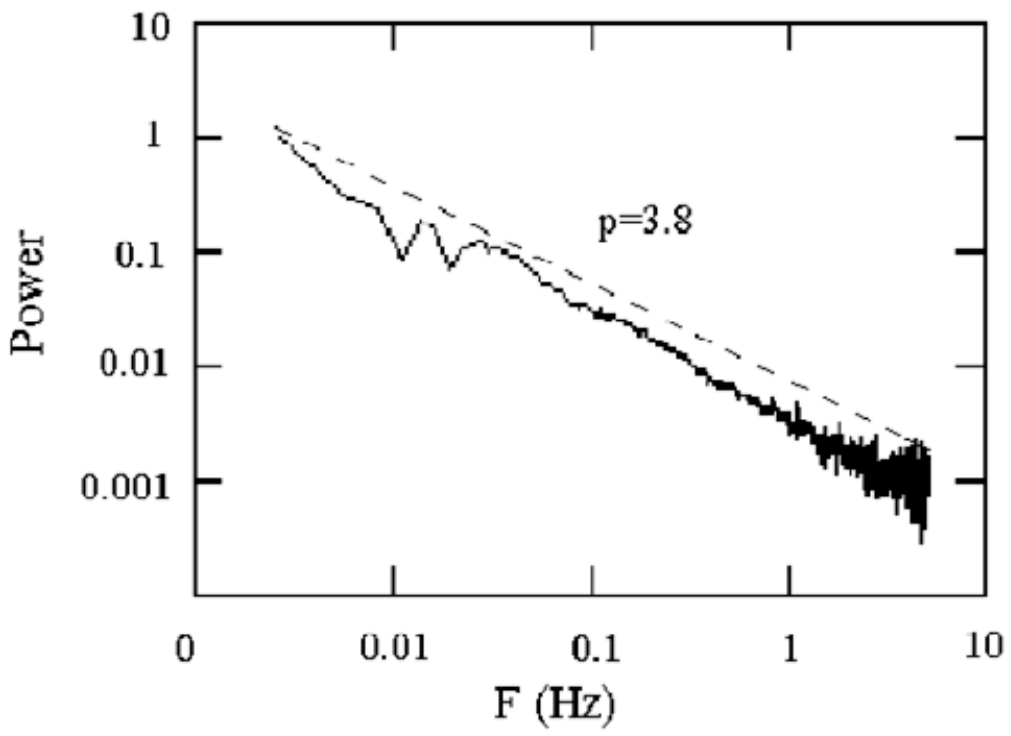

Figure 1.

Our calculations, particularly, demonstrated, that structure of interferometer power spectrum depends on intensity of emission fluctuations, induced by turbulent medium. At case of weak phase fluctuations it is possible to determine the index of spatial spectrum of electron density fluctuations; if the interferometer baseline is oriented mainly along the electron density irregularities transfer, the solar wind velocity are estimated. At strong phase disturbances power spectrum has got a Gaussian form independently of spatial spectrum of electron density distribution. In that case the spectrum width carries information about intensity of phase fluctuations.

Obtained experimental power spectra let us to get estimations of index of spatial spectrum $p$. During observations $\mathrm{p}$ took on a value 3.2-4.5 depending on the angular distances of radio sources to the Sun.

The sample of power spectrum at weak phase fluctuations is displayed on fig. 1 . This spectrum obtained at observation of source NRAO530, located at angular distance from the Sun (=16 degrees (baseline Bear Lakes (RT-64, Russia) - Noto (RT-32, Italy), wavelength $18 \mathrm{~cm}$, integration time $t=0.1 \mathrm{sec}, 4$ December, 1999). Spectral index p, evaluated on the slope of power spectrum, is equal to 3.8 .

This work is supported by grant RFBR No 02-02-39023, NSCF No 10173015.

\section{References}

Gavrilenko, V. G., Lipatov, B. N. \& Nechaeva, M. B. 2002 Radiophysics and Quantum Electronics 45, 419-430.

Alimov, V. A., Gavrilenko, V. G., Lipatov, B. N. \& Nechaeva, M. B. 2004 Radiofizika [in Russian] 47, 167-179. 